\title{
Robust Stability of Polytopic-Type Uncertain Singular Stochastic Systems with Time-Varying Delays
}

\author{
Yuanqiang Chen \\ College, Guizhou Minzu University, Guiyang, 550025, China
}

\begin{abstract}
The problems of robust stability for a class of the polytopic-type uncertain singular stochastic systems with time-varying delays are studied. By using a delay decomposition approach and terms of linear matrix inequalities (LMIs), robust stability criteria ensuring globally stochastically asymptotic stability of the polytopic-type uncertain singular stochastic systems with time-varying delays are established. New globally stochastically asymptotic stable criteria for the certain stochastic systems with discrete and distributed delays are obtained in terms of linear matrix inequalities and Leibniz-Newton formula as well. Finally, the validity of the obtained results is shown by a numerical example.
\end{abstract}

Keywords-singular systems; stochastic systems; polytopic-type uncertainties; delayed decomposition approach; linear matrix inequalities

\section{INTRODUCTION}

Time delay are frequently encountered in various areas, including engineering, biology, and economics. Time delay arises naturally in connection with system process and information flow for different part of dynamical systems. A time delay is often a source of instability and oscillations in a system. Therefore, considerable efforts are concentrated on the stability analysis for systems including time delays. Thus, the problem of stability analysis for time-delay systems has been an important topic [1-3] and references therein. In the past few years, the robust stability of uncertain systems with time delays has received considerable attention; and many papers have focused on time-delay systems with polytopic-type uncertainties. On the other hand, due to the much more conservatism of delay-independent conditions compared with delay-dependent ones when time-delay is small, chasing delay-dependent stability criteria is of much theoretical and practical value. Current efforts to achieve robust stability in time-delay systems can be divided into two categories [4], namely delay-independent criteria [5] and delay-dependent criteria [6-8]. Recently, in order to reduce conservatism, Park [9] presented an improved version of the standard bounding method and obtained some delay-dependent criteria for linear time-delay systems that were better than previous results. Fridman [10] proposed a descriptor model transformation of the original system into an equivalent one. Though some less conservative delay-dependent criteria are obtained, the conservatism still remains due to the fact that it used the bounding technique for cross terms. Fridman and Shaked [7]combined Park's and Moon's inequalities with a descriptor model transform and obtained more efficient criteria for systems with polytopic-type uncertainties. Since it does not employ bounding techniques for cross terms, this approach can lead to less conservative results. However, even though these efforts have produced great progress, some issues remain that require reconsideration. To give an example, when the upper bound of delay derivative may be larger than or equal to 1 , Zhu and Yang [10] used a delay decomposition approach and used the Leibniz-Newton formula. There must be a relationship between them; but this point was not considered.

Many results have been established for singular systems with time-delay which can be classified into two types, delay-independent condition and delay-dependent condition [11-14]. Most of the research results have focused on delay-dependent conditions for the less conservatism. Such as, in [15], a delay-range-dependent condition for unforced singular systems with multiple time-varying delays was given to ensure the system to be regular, impulse-free and $\alpha$-stable, then, a method to estimate the system's convergence rate was proposed. To the author's knowledge, the research on the stochastic singular system with multiple time-varying delays is rather limited, because the study of singular system with multiple time-varying delays is much more complicated than the singular system with single time-delay or without time-delay, it is due to the difficulty of guaranteeing the stability of the fast subsystem [16].

This paper presents some robust stability criteria for more complex system involving the derivative of the delayed state. First, based on the Lyapunov function method, for polytopic-type uncertain singular stochastic systems with time-varying delays, a less conservative criterion is obtained By using a delay decomposition approach and terms of linear matrix inequalities (LMIs). Moreover, for certain stochastic systems with discrete and distributed, we propose new stability criteria in terms of linear matrix inequalities and Leibniz-Newton formula, which are less conservative than delay-independent one when the size of delays is small. Our results will be compared with those developed in the recent literature to show that they are less conservative. Numerical example shows that the results obtained in this note are effective and are an improvement over existing criteria.

\section{PRELIMINARIES}

In this section, we introduce some preliminary concepts which will be found useful in the paper. Consider the following polytopic-type uncertain singular stochastic systems with time-varying delays 


$$
\begin{aligned}
& E \mathrm{~d} x(t)=\left[A_{j} x(t)+B_{j} x(t-h(t))\right] \mathrm{d} t+\sigma(t, x(t), x(t-h(t))) \mathrm{d} \omega(t), t \geq 0, \\
& x(t)=\phi(t), t[-h, 0], j=1,2, \cdots, m
\end{aligned}
$$

where $x(t) \in R^{n}$ is the state vector. The matrices $A_{j}, B_{j}$ are constant matrices with appropriate dimensions, and satisfy the real convex polytopic model[17]

$$
\Omega=\left\{[A, B]=\sum_{j=1}^{n} \xi_{j}\left[A_{j}, B_{j}\right]: \sum_{j=1}^{n} \xi_{j}=1, \xi_{j} \geq 0\right\},
$$

where $A, B$ are constant matrices with appropriate dimensions and $\xi_{j}(j=1,2, \cdots, m)$ are time-invariant uncertainties. $\omega(t)$ is a Brownian motion defined on a complete probability space $(\Omega, F, P)$ with a natural filtration $\{F\}_{t \geq 0}$. Let $\sigma(t, x(t), x(t-h(t))) \mathrm{d} \omega(t)$ is locally Lispchitz continuous and satisfies the linear growth condition as well, where $\sigma(t, 0,0)=0 . \phi(t)$ is a smooth vector-valued initial function; $h(t)$ is a time-varying delay in the state, and $h$ is an upper bound on the delay $h(t)$.

Without loss of generality, we assume that

$$
\begin{gathered}
x(t)=\left[\begin{array}{l}
X_{1}(t) \\
x_{2}(t)
\end{array}\right], E=\left[\begin{array}{cc}
I_{r} & 0 \\
0 & 0
\end{array}\right], A_{j}=\left[\begin{array}{ll}
A_{1 j} & A_{2 j} \\
A_{3 j} & A_{4 j}
\end{array}\right], B=\left[\begin{array}{ll}
B_{1 j} & B_{2 j} \\
B_{3 j} & B_{4 j}
\end{array}\right], \\
\operatorname{trace}\left[\sigma^{T}(t, x, y) P \sigma(t, x, y)\right] \leq x(t)^{T} D_{1} x(t)+y^{T} D_{2} y,
\end{gathered}
$$

where $x_{1}(t) \in R^{r}, A_{1 j} \in R^{r \times r}, B_{1 j} \in R^{r \times r}, D_{1}, D_{2}$ and $P$ are positive-definite matrices with appropriate dimensions.

To get the main results of this paper, the following preliminary lemmas are needed.Define the difference operator $\Lambda$ :

$$
\Lambda\left(x_{2}(t)\right)=x_{2}(t)+A_{4_{j}}^{-1} B_{4 j} x_{2}(t-h(t)) .
$$

Lemma 1 Consider the unforced singular time-delay system

$$
E \dot{x}(t)=A_{j} x(t)+B_{j} x(t-h(t))
$$

with $E, A_{j}, B_{j}$ given in (3). If the operator $\Lambda$ in (4) is stable and there exist positive numbers $\alpha, \beta, \gamma$ and a continuous functional $V$ such that

$$
\alpha\left\|x_{1}(t)\right\|^{2} \leq V(x(t)) \leq \beta\|x(t)\|^{2}, \dot{V}(x(t)) \leq-\gamma\|x(t)\|^{2},
$$

and the function $V(x(t))$ is absolutely continuous for $x(t)$ satisfying (5), then (5) is asymptotically stable.

Lemma 2 ([18]) For any positive semi-definite matrices

$$
X=\left[\begin{array}{ccc}
X_{11} & X_{12} & X_{13} \\
* & X_{22} & X_{23} \\
* & * & X_{33}
\end{array}\right] \geq 0
$$

the following integral inequality holds

$$
\begin{aligned}
& -\int_{t-h(t)}^{t} \dot{x}(s)^{T} X_{33} \dot{x}(s) d s \leq \int_{t-h(t)}^{t}\left[x(t)^{T} x(t-h(t))^{T} \dot{x}(s)^{T}\right] \\
& {\left[\begin{array}{ccc}
X_{11} & X_{12} & X_{13} \\
* & X_{22} & X_{23} \\
* & * & 0
\end{array}\right]\left[\begin{array}{c}
x(t) \\
x(t-h(t)) \\
\dot{x}(s)
\end{array}\right] d s}
\end{aligned}
$$

\section{MAin Results}

In this section, this paper finds new stability criteria less conservative than the existing results. For polytopic-type uncertain singular stochastic systems (1) with time-varying delays, we shall first give robust condition by using a delay decomposition approach as follows. Second, we consider the case of certain stochastic systems with discrete and distributed delays.

The result on the robust stability of polytopic-type uncertain singular stochastic systems (1) with time-varying delays is presented in the following theorem.

Theorem 1 Let $h(t)$ be a differentiable function, satisfying $h^{\prime}(t) \leq \mu<1, t \geq 0$, the polytopic-type uncertain singular stochastic systems (1) with time-varying delays is globally robustly stochastically asymptotic stable if there exist matrices $P>0$ and $G>0, Q_{j}>0, R_{j} \geq 0, W_{j} \geq 0$ and $Y_{j}$ $(j=1,2, \cdots, m)$, and positive semi-definite matrices

$$
X^{(j)}=\left[\begin{array}{ccc}
X_{11}^{(j)} & X_{12}^{(j)} & X_{13}^{(j)} \\
* & X_{22}^{(j)} & X_{23}^{(j)} \\
* & * & X_{33}^{(j)}
\end{array}\right] \geq 0,(j=1,2, \cdots, m),
$$

such that

$$
\tilde{G}=\left[\begin{array}{cc}
G & \tilde{Y} \\
* & (1-\mu) \tilde{W}-X_{33}
\end{array}\right] \geq 0, \Theta=\left[\begin{array}{cccc}
\Theta_{11} & \Theta_{12} & 0 & 0 \\
* & \Theta_{22} & 0 & 0 \\
* & * & \Theta_{33} & 0 \\
* & * & * & \Theta_{44}
\end{array}\right]<0 .
$$

where

$$
\begin{gathered}
\Theta_{11}=A^{T} P+P A+Y+Y^{T}+R+D_{1}+h\left(X_{11}+G\right)+X_{13}+\left(X_{13}\right)^{T}, \\
\Theta_{12}=P B-Y+h X_{12}-X_{13}+\left(X_{23}\right)^{T}, \\
\Theta_{22}=D_{2}+h X_{22}-X_{23}-\left(X_{23}\right)^{T}-(1-\mu) R, \Theta_{33}=\tilde{Q}+h \tilde{W}, \\
\Theta_{44}=-\tilde{Q}, \tilde{Y}=[Y, 0], \tilde{Q}=\left[\begin{array}{ll}
Q & 0 \\
0 & 0
\end{array}\right], \tilde{W}=\left[\begin{array}{cc}
W & 0 \\
0 & 0
\end{array}\right], \\
Y=\sum_{j=1}^{m} \xi_{j} Y_{j},
\end{gathered}
$$




$$
\begin{gathered}
R=\sum_{j=1}^{m} \xi_{j} R_{j}, Q=\sum_{j=1}^{m} \xi_{j} Q_{j}, W=\sum_{j=1}^{m} \xi_{j} W_{j}, \\
X_{i k}=\sum_{j=1}^{m} \xi_{j} X_{i k}^{(j)}(i, k=1,2,3) .
\end{gathered}
$$

Now, we consider the case in which the matrices $A_{j}$ and $B_{j}$ are fixed, i.e., Consider the following certain stochastic systems with discrete and distributed delays

$$
\begin{aligned}
& \mathrm{d} x(t)=[A x(t)+B x(t-h(t))+C \dot{x}(t-h)] \mathrm{d} t \\
& +\sigma(t, x(t), x(t-h(t))) \mathrm{d} \omega(t), t \geq 0, \\
& x(t)=\phi(t), t[-h, 0] .
\end{aligned}
$$

When $C=0$, by Theorems 2 , it is easy to derive the following Corollary.

Corollary 1 Let $h(t)$ be not differentiable or the upper bound of the derivative of $h(t)$ is unknown, the certain stochastic system (8) with time-varying delays is globally stochastically asymptotic stable if there exist matrices $P=P^{T}>0, Q=Q^{T}>0, R=R^{T} \geq 0, W=W^{T} \geq 0$, positive semi-definite matrices

$$
X=\left[\begin{array}{cccc}
X_{11} & X_{12} & X_{13} & X_{14} \\
* & X_{22} & X_{23} & X_{24} \\
* & * & X_{33} & X_{34} \\
* & * & * & X_{44}
\end{array}\right] \geq 0,
$$

and appropriately dimensioned matrices $N_{i}(i=1,2,3,4)$, such that

$$
\begin{aligned}
\mathrm{T} & =\left[\begin{array}{ccccc}
X_{11} & X_{12} & X_{13} & X_{14} & N_{1} \\
* & X_{22} & X_{23} & X_{24} & N_{2} \\
* & * & X_{33} & X_{34} & N_{3} \\
* & * & * & X_{44} & N_{4} \\
* & * & * & * & W
\end{array}\right] \geq 0, \\
\Xi & =\left[\begin{array}{ccccc}
\Xi_{11} & \Xi_{12} & \Xi_{13} & \Xi_{14} & 0 \\
* & \Xi_{22} & \Xi_{23} & \Xi_{24} & 0 \\
* & * & \Xi_{33} & \Xi_{34} & 0 \\
* & * & * & \Xi_{44} & 0 \\
* & * & * & * & \Xi_{55}
\end{array}\right]<0,
\end{aligned}
$$

where

$$
\begin{gathered}
\Xi_{11}=A^{T} P+P A+N_{1}+N_{1}^{T}+h X_{11}+D_{1}, \\
\Xi_{12}=P B-N_{1}-N_{2}+h X_{12}, \Xi_{13}=N_{3}+h X_{13}, \\
\Xi_{14}=N_{4}+h X_{14}, \Xi_{22}=-N_{2}-N_{2}^{T}+h X_{22}+D_{2}, \\
\Xi_{23}=-N_{3}+h X_{23}, \Xi_{24}=-N_{4}+h X_{24}, \\
\Xi_{33}=Q+h W+h X_{33}, \Xi_{34}=h X_{34}, \Xi_{44}=-Q+h X_{44}, \\
\Xi_{55}=-R .
\end{gathered}
$$

\section{NUMERICAL EXAMPLE}

In this section, we will present a numerical example to illustrate the usefulness of our main results. Consider the following the certain stochastic system(9) with discrete and distributed delays as follows

$$
\begin{gathered}
\mathrm{d} x(t)=\left[\begin{array}{cc}
-1.7073 & 0.6856 \\
0.2279 & -0.6368
\end{array}\right] x(t)+\left[\begin{array}{cc}
-2.5026 & -1.0540 \\
-0.1856 & -1.5715
\end{array}\right] x(t-h(t)), \\
\left.+\left[\begin{array}{cc}
0.0558 & 0.0360 \\
0.2747 & -0.1084
\end{array}\right] \dot{x}(t-h)\right] \mathrm{d} t+\sigma(x, y) \mathrm{d} \omega(t) \\
\text { with initial state }[-1,1]^{T}, h=3 \text { and } \mu=0 \text {,and } \\
\sigma(x, y)=\sqrt{0.05} \operatorname{diag}\left\{x_{1} \sin \left(x_{1} y_{1}\right)+\right. \\
\left.x_{2} \sin \left(x_{2} y_{2}\right), x_{1} \cos \left(x_{1} y_{1}\right)+x_{2} \cos \left(x_{2} y_{2}\right)\right\}
\end{gathered}
$$

It is easy to calculate that $D_{1}=D_{2}=\sqrt{0.1} I$.

We verify that all the conditions of Theorem 2 are satisfied. Thus, certain stochastic system (9) with discrete and distributed delays is globally stochastically asymptotic stable, and its state chart is shown in Figure.1 below. It is clear that the state of system (9) reaches equilibrium point after 55 second.

When $C=0$, we verify that all the conditions of Corollary 1 are satisfied. Thus, the certain stochastic system (9) with time-varying delays is globally stochastically asymptotic stable, and its state chart is shown in Figure. 2 below. It is clear that the state of system (9) reaches equilibrium point after 25 second.

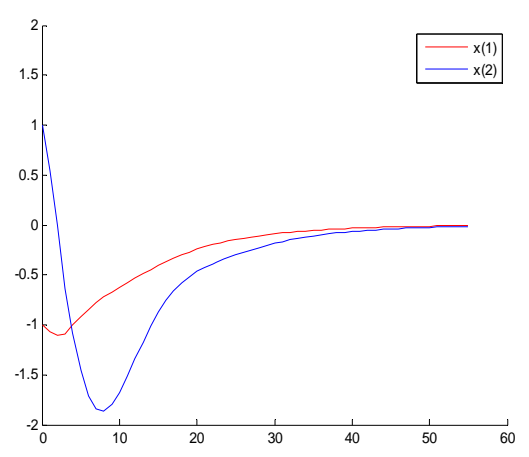

FIGURE I. THE TIME SEQUENCE CHART WHEN $h=3$ AND $\mu=0$

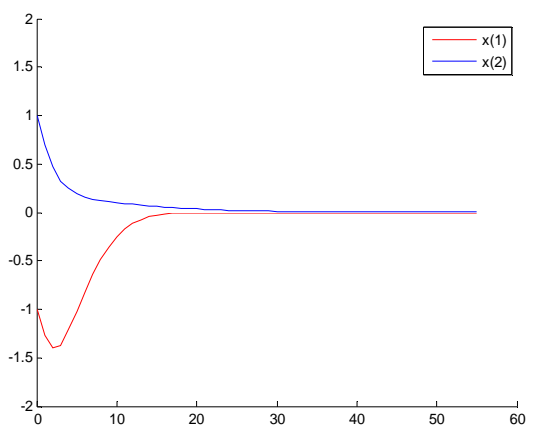

FIGURE II. THE TIME SEQUENCE CHART WHEN $h=3, \mu=0$ AND $C=0$ 


\section{CONCLUSION}

This note presents some new robust stability criteria for the polytopic-type uncertain singular stochastic systems with time-varying delays. New techniques were developed to make the criteria less conservative. First, globally robustly stochastically asymptotic stable condition are obtained by using a delay decomposition approach and terms of linear matrix inequalities (LMIs), which can be easily solved by various optimization algorithms. Then, a globally stochastically asymptotic stable criterion for certain stochastic system with discrete and distributed delays was derived by considering the relationships among the terms in the system variables. The relationships among the terms in the Leibniz-Newton formula were also taken into account using some free weighting matrices, which were selected by LMIs. A numerical example demonstrates the validity of these methods. The results show that the methods described in this note are very effective.

\section{ACKNOWLEDGEMENT}

This work was partially supported by the Natural Science Foundation of Guizhou Province under Grant [2014]2089, and the National Natural Science Foundation of China under Grant 11171079, and the Mathematical Modeling \& Application Talents Team of Guizhou Provinc under Grant [2013]405.

\section{REFERENCES}

[1] P. Balasubramaniam, R. Krishnasamy, R. Rakkiyappan, Delay-dependent stability of neutral systems with time-varying delays using delay-decomposition approach. Appl. Math. Model. 36 (2012) 2253-2261.

[2] P.L. Liu, Stability criteria for neutral uncertain systems with time-varying delays. J. Syst. Contr. Eng. 224 (4) (2010) 339-348.

[3] X.L. Zhu, G.H. Yang, New results of stability analysis for systems with time-varying delay. Int. J. Robust Nonlinear Control 20 (2010) 596-606.

[4] T. Mori, Criteria for asymptotic stability of linear time-delay systems. IEEE Trans. Automat. Contr., 30(1985) 158-161.

[5] J. H. Lee, S. W. Kim, and W. H. Kwon, Memory less H controllers for state delayed systems. IEEE Trans. Automat. Contr., 39(1994) 159-162.

[6] Y. Q. Xia and Y. M. Jia, Robust stability functional of state delayed systems with polytopic type uncertainties via parameter-dependent Lyapunov functions. Int. J. Control, 75(16)(2002)1427-1434.

[7] E. Fridman and U. Shaked, An improved stabilization method for linear time-delay systems. IEEE Trans. Automat. Contr., 47(2002)1931-1937.

[8] Q. L. Han, New results for delay-dependent stability of linear systems with time-varying delay. Int. J. Syst. Sci., 33(2002) 213-228.

[9] P. Park, A delay-dependent stability criterion for systems with uncertain time-invariant delays. IEEE Trans. Automat. Contr., 44(1999) 876-877.

[10] E. Fridman, New Lyapunov-Krasovskii functionals for stability of linear retarded and neutral type systems. Syst. Contr. Lett. 43 (2001) 309-319.

[11] J. Li, H. Su, Y. Zhang, Z. Wu, J. Chu, Chattering free sliding mode control for uncertain discrete time-delay singular systems. Asian J. Control, 15(1) (2013)260-269.

[12] J. Li, H. Su, Z. Wu, J. Chu, Less conservative robust stability criteria for uncertain discrete stochastic singular systems with time-varying delay. Int. J. Syst. Sci. 44(3) (2013) 432-441.

[13] S. Ma, C. Zhang, $\mathrm{H} \infty$ control for discrete-time singular Markov jump systems subject to actuator saturation. J. Frankl. Inst. 349 (2012)1011-1029.

[14] Z. Wu, J.H. Park, H. Su, J. Chu, Stochastic stability analysis for discrete-time singular Markov jump systems with time-varying delay and piecewise-constant transition probabilities. J. Frankl. Inst. 349(2012)2889-2902.
[15] A. Haidar, E.K. Boukas, Exponential stability of singular systems with multiple time-varying delays. Automatica, 45 (2009)539-545.

[16] F.A. Khasawneh, B.P. Mann, A spectral element approach for the stability analysis of time-periodic delay equations with multiple delays. Commun. Nonlinear Sci. Numer. Simul. 18(2013) 2129-2141.

[17] Y. He, M. Wu, J.H. She, G. P. Liu, Parameter-Dependent Lyapunov functional for stability of time-delay systems with polytopic-type uncertainties. IEEE Transactions on Automatic Control, 49 (5)(2004) 828-832.

[18] P.L. Liu, A delay decomposition approach to stability analysis of neutral systems with time-varying delay. Applied Mathematical Modelling 37 (2013) 5013-5026. 